\title{
Malária Grave Importada. Relato de Caso*
}

\author{
Severe Imported Malaria. Case Report
}

\author{
Alessandra Alves ${ }^{1}$, Andréa Martins², Samantha Adolphsson ${ }^{3}$, Bruno Bockorny ${ }^{4}$, \\ Guilherme Carleti ${ }^{4}$, Gustavo Cabral ${ }^{4}$, Antônio Carlos P. Souza ${ }^{5}$, Arthur Vianna ${ }^{6}$
}

\section{RESUMO}

JUSTIFICATIVA E OBJETIVOS: A malária ainda representa um problema de saúde global. A forma grave da doença é causada principalmente por $P$. falciparum e pode cursar com complicações cerebrais, renais, pulmonares, hematológicas, circulatórias e hepáticas. O objetivo deste estudo foi relatar um caso de paciente portador de malária grave importada.

RELATO DO CASO: Paciente do sexo masculino, 30 anos, pardo, filipino, marinheiro, proveniente de embarcação vinda da Nigéria, com história de dor abdominal no hipocôndrio direito, icterícia, febre e rebaixamento do nível de consciência. Os exames laboratoriais de admissão mostraram hiperbilirrubinemia de $50 \mathrm{mg} / \mathrm{dL}$, acidose metabólica grave, trombocitopenia, creatinina de $5,6 \mathrm{mg} / \mathrm{dL}$, leucocitose com desvio até metamielócitos. O escore APACHE II foi de 37, com risco de óbito de $88 \%$. Durante a internação foi diagnosticada malária por $P$. falciparum pelo teste de gota espessa. Mes-

1. Médica Rotina do CTI da Clínica São Vicente, Rio de Janeiro; Médica Especializada em Medicina Intensiva pela UFRJ.

2. Médica Rotina do CTI da Clínica São Vicente, Rio de Janeiro, Título de Especialista em Medicina Intensiva pela AMIB.

3. Graduanda em Medicina pela UNIRIO; Estagiária do CTI da Clínica São Vicente, Rio de Janeiro.

4. Graduando em Medicina pela UFRJ; Estagiário do CTI da Clínica São Vicente, Rio de Janeiro.

5. Médico Assistente, Especializado em Clínica médica

6. Médico Coordenador do CTI da Clínica São Vicente, Rio de Janeiro; Título de especialista em Medicina Intensiva pela AMIB.

*Recebido do Centro de Terapia Intensiva da Clínica São Vicente, Rio de Janeiro, RJ

Apresentado em 12 de abril de 2007

Aceito para publicação em 31 de maio de 2007

Endereço para correspondência:

Dra. Alessandra Alves

Rua João Borges, 204 - Gávea

22451-100 Rio de Janeiro, RJ

Fone: (21) 2529-4487

E-mail: ale76@terra.com.br

(C)Associação de Medicina Intensiva Brasileira, 2007 mo com tratamento antimalárico adequado, o paciente evoluiu com insuficiência renal aguda necessitando de hemodiálise e síndrome de angústia respiratória aguda (SARA), necessitando de ventilação mecânica (VM), choque refratário tratado com aminas vasoativas, além de quadro hematológico, configurando um caso grave de disfunção de múltiplos de órgãos. Ainda apresentou pneumonia associada à VM e sepse relacionada ao uso de cateteres. Após a alta hospitalar, o paciente não apresentou seqüelas cerebral, pulmonar ou renal.

CONCLUSÕES: Dos critérios definidores de malária grave descritos na literatura, o paciente preenchia: insuficiência renal aguda, síndrome da angústia respiratória aguda (SARA), acidose metabólica, alteração do nível de consciência, hemoglobinúria macroscópica, hiperparasitemia e hiperbilirrubinemia, que se relaciona a uma mortalidade maior que $10 \%$, na dependência do tratamento precoce e dos recursos disponíveis. A malária grave exige diagnóstico e tratamento intensivo rápidos, pois o atraso aumenta a morbimortalidade do paciente.

Unitermos: malária grave, malária grave importada, $P$. falciparum, terapia antimalárica

\section{SUMMARY}

BACKGROUND AND OBJECTIVES: Malaria is still considered a major global health problem. The severity form of the disease is caused, mainly by $P$. falciparum and may occur together with cerebral, kidney, pulmonary, hematologic, circulatory and hepatic complications. This report is about a patient with a case of severe imported malaria.

CASE REPORT: A 30-years-old man, mulatto, Philippine, sailor, coming from a ship arriving from Nigeria, with a history of abdominal pain on the right hypochondrium, jaundice, fever, decreased in the consciousness. Lab tests made upon his admission showed hyperbilirubinemia at a level of $50 \mathrm{mg} / \mathrm{dL}$, severe metabolic acidosis, thrombocytopenia, creatinine levels of $5.6 \mathrm{mg} / \mathrm{dL}$ and leukocytosis with deviation through metamyelocytes. 
The APACHE II score was 37, with death estimated risk of $88 \%$. During his stay at the hospital, P. Falciparum Malaria was diagnosed through the thick drop test. And, even with the adequate anti-malaria therapy, the patient's condition evolved to an acute renal failure requiring hemodialis; acute respiratory distress syndrome (ARDS); septic shock, and hematological disorders, forming a multiple organ dysfunction syndrome (MODS). After being discharged from the hospital, the patient did not present any cerebral, pulmonary or kidney sequel.

CONCLUSIONS: From the criteria described in medical literature to define critical malaria, the patient fulfilled the following: acute renal failure, ARDS, metabolic acidosis, altered level of consciousness, macroscopic hemoglobinuria, hyperparasitism and hyperbilirubinemia, related to a lethality rate of over $10 \%$, depending on early treatment and available resources. Severe malaria requires fast diagnosis allied to a quick access to an intensive care treatment, since any delay increases the morbid-mortality of the disease.

Key Words: anti-malaria therapy, $P$. falciparum, severe malaria, severe imported malaria.

\section{INTRODUÇÃO}

A malária é uma doença infecto-parasitária que pode se apresentar de forma grave, sobretudo quando causada pelo $P$. falciparum, cursando com diversas disfunções sistêmicas. A mortalidade pode ser maior que $10 \%$, dependendo do tratamento precoce e dos recursos disponíveis.

O objetivo deste estudo foi relatar um caso de paciente portador de malária grave importada, cujo escore APACHE II na admissão foi de 37, com risco de óbito de $88 \%$, denotando a gravidade da doença. Apesar da gravidade do quadro e da ocorrência de diversas complicações algumas inerentes a evolução gravíssima apresentada pelo paciente e sem relação direta com o plasmodium, o paciente recebeu o tratamento antimalárico conforme preconizado pelo Ministério da Saúde e evoluiu sem seqüelas, sendo o suporte intensivo dispensado ao paciente de extrema importância para o desfecho.

\section{RELATO DO CASO}

Paciente do sexo masculino, 30 anos, pardo, filipino, marítimo, chegou na emergência da Clínica São Vicente no dia 18 de outubro proveniente de embarcação vinda da Nigéria, apresentando febre alta intermitente havia sete dias, dor abdominal no hipocôndrio direito e icterícia, evoluindo com piora do quadro e diminuição do nível de consciência, febril, piora da dor abdominal e distensão. Foi realizada profilaxia para malária conforme protocolo usado pelo navio $(250 \mathrm{mg}$ de fosfato de cloroquina por semana).

Foi admitido no Serviço de Emergência hemodinamicamente estável e torporoso. Realizada tomografia computadorizada de abdômen (TC) evidenciando hepatomegalia, com vesícula contraída e ausência de líquido livre na cavidade peritoneal. TC de crânio e tórax normais.

Foi transferido para o centro de terapia intensiva (CTI) deste mesmo hospital. O exame inicial revelou um paciente torporoso, não responsivo a solicitações verbais, movimentando ativamente o dimídio direito, parético em dimídio esquerdo e apresentando desvio do olhar conjugado para a direita. Pupilas isofotorreagentes. Apresentava-se hipocorado $(2+/ 4+)$, ictérico $(4+/ 4+)$, taquipnéico, hipotenso (PA: $80 \times 40 \mathrm{mmHg}$ ), taquicárdico e anúrico. $O$ restante do exame se apresentava normal.

Os dados laboratoriais no CTI apresentavam-se da seguinte forma: hematócrito: 26,9\%; hemoglobina: 9,1 $\mathrm{mg} / \mathrm{dL}$; leucócitos: 17.500 , com desvio até metamielócitos; plaquetas: 19000; TAP e PTT normais; uréia: 187; creatinina: 5,6; bilirrubina total: 50 ; bilirrubina direta: 38 ; proteínas totais: 6; albumina: 2,6; TGO: 203; TGP: 300; fosfatase alcalina: 189; gama-GT: 89; lactato: 10,8; glicemia: $64 \mathrm{mg} / \mathrm{dL}$; gasometria arterial: $\mathrm{pH}$ : 7,18; $\mathrm{pCO}_{2}$ : 22; $\mathrm{pO}_{2}$ : 115; P/F: 383; $\mathrm{HCO}_{3}$ : 7,8; BE: -18,9; $\mathrm{SaO}_{2}$ : 96,3\%; $\mathrm{SvO}_{2}: 76 \%$. O escore APACHE II calculado foi de 37 , com risco de óbito de $88 \%$.

O paciente foi intubado, puncionado acesso venoso profundo e linha arterial. Recebeu transfusão de plaquetas, reposição volêmica, iniciada noradrenalina e sedação com midazolam em infusão contínua. Após 5 horas de tratamento, o paciente apresentava-se com febre contínua, elevação de escórias nitrogenadas, anúrico, dependente de aminas, tendo sido iniciado suporte dialítico continuo. Devido à história epidemiológica e forte suspeita clínica, foi coletado material para exame da gota espessa e iniciado empiricamente a administração de artesunato, por via venosa, que permaneceu por seis dias. A análise do sangue evidenciou a presença de mais do que 100.000 plasmódios por $\mathrm{mm}^{3}$ de sangue periférico, sendo eles $P$. falciparum. Após três dias de tratamento com artesunato, foi iniciado tratamento com clindamicina, por via venosa, mantido por 7 dias. 
Após 2 dias de internação no CTI, o paciente apresentou disfunções orgânica de múltiplos órgãos, incluindo renal, respiratória, cardiovascular e hematológica. Apresentava piora clínica e elevação da bastonemia, e da proteína C-reativa (PCR). A radiografia de tórax revelou condensações heterogêneas nos $2 / 3$ inferiores de ambos os pulmões, sobretudo à direita. Além disso, evidenciou piora troca gasosa, com relação da pressão arterial de oxigênio e fração inspirada de oxigênio (P/F) de 175 e ecocardiograma com funções sistólica e diastólica preservadas, sustentando o diagnóstico de síndrome da angústia respiratória aguda (SARA). Realizou-se lavado broncoalveolar (LBA) sendo iniciado tratamento empírico com piperacilina-tazobactan para pneumonia associada à ventilação mecânica, sendo submetido também a manobras de recrutamento alveolar, resultando em melhora da troca gasosa.

Nos dias seguintes da internação manteve-se grave e dependente de aminas. Apresentou um episódio de sangramento oral e a endoscopia digestiva alta revelou gastrite com sangramento ativo, tendo sido aumentada a dose do bloqueador de bomba de prótons, com melhora evolutiva.

No $9^{\circ}$ dia de internação, evoluiu com aumento dos níveis de amilase e lípase, 366 e 1475, respectivamente, associada à distensão abdominal. Realizada nova TC de abdômen que evidenciou edema pancreático com gordura peri-pancreática preservada, confirmando o diagnóstico de pancreatite aguda. Diante da intolerância a progressão da dieta enteral foi então iniciada nutrição parenteral.

No $11^{\circ}$ dia, o cateter venoso profundo foi substituído, pois o paciente mantinha episódios febris apesar de melhora clínica evolutiva. No entanto posteriores resultados microbiológicos não confirmaram a suspeita de infecção associada ao cateter.

No dia $18^{\circ}$ dia, diante da progressiva melhora clínica e laboratorial foi procedida a extubação com sucesso, necessitando somente de suporte ventilatório não-invasivo intermitente.

Após 22 dias de internação instalou-se novo quadro febril, com piora hemodinâmica e laboratorial (14.400 leucócitos com $10 \%$ bastões na periferia). Foram realizadas hemocultura, urinocultura e cultura da ponta de cateter venoso profundo, sendo isolada Klebsiella pneumoniae produtora de $\beta$-lactamase de espectro ampliado (ESBL) nos dois últimos materiais. Então foi iniciado ertapenem com boa resposta terapêutica.

Após 24 dias de internação recebeu alta do CTI hemodinamicamente estável, ventilando em ar ambiente, sem déficits neurológicos e em diálise intermitente, recebendo antibiótico para tratamento da infecção associada à cateter venoso profundo.

\section{DISCUSSÃO}

A malária é uma doença infecto-parasitária caracterizada por ciclos de febre e calafrios associados à lise sincrônica de hemácias parasitadas. Os agentes etiológicos são protozoários intraeritrocíticos obrigatórios do gênero Plasmodium ( $P$. falciparum, $P$. malariae, $P$. vivax e $P$. ovale). A transmissão pode ser feita através da picada de mosquito anofelino fêmea infectado, por transfusão sanguínea ou transmissão congênita. A gravidade e as manifestações clínicas características da malária dependem da espécie infectante, da magnitude da parasitemia, dos efeitos metabólicos do parasita e das citocinas liberadas. A maioria dos casos de malária grave e de óbitos é causada pelo $P$. falciparum.

Nos países industrializados, a maioria dos casos de malária ocorre entre viajantes, imigrantes ou militares que retornam de áreas endêmicas da doença, a chamada malária importada. Cerca de $5 \%$ dos casos de malária importada correspondem à malária grave ${ }^{1}$. A sua letalidade pode ser superior a 10\%, na dependência do tratamento precoce e dos recursos disponíveis. Atualmente, cepas de $P$. falciparum resistentes aos agentes antimaláricos continuam disseminando-se pelo mundo, o que torna o combate à doença mais difícil. Resistência à cloroquina ou à sulfadoxina-pirimetamina é encontrada em praticamente todas as áreas de transmissão da malária. Na América do Sul e Sudeste Asiático concentram-se os focos de $P$. falciparum multiresistentes (resistência a duas ou mais classes de fármacos) $)^{2}$.

O período de incubação da doença é cerca de 10 a 14 dias, podendo ser maior em indivíduos que já foram expostos previamente, que já usaram antimaláricos de forma profilática, que foi o caso do paciente relatado, ou que já trataram parcialmente a infecção. A apresentação clínica da malária assemelha-se a infecções virais comuns, o que pode atrasar o correto diagnóstico da doença. A maioria dos pacientes apresenta febre, calafrios, cefaléia e diaforese ${ }^{1}$. Outros sintomas incluem: mal-estar, náusea, vômitos, mialgia, dor abdominal, diarréia leve e tosse seca. Taquicardia, icterícia, palidez, hipotensão ortostática e hepato-esplenomegalia são sinais que também podem ser encontrados nesses pacientes. Com relação aos achados laboratoriais, trombocitopenia, hiperbilirrubinemia, anemia e 
elevação de transaminases hepáticas são as alterações mais comumente encontradas ${ }^{3}$. O leucograma é normal, havendo neutrofilia com desvio à esquerda na maioria dos casos. O VHS, os níveis de proteína Creativa e procalcitonina quase sempre estão aumentados.

Em relação à malária grave, existem critérios clínicos e laboratoriais estabelecidos pela Organização Mundial de Saúde (OMS), que funcionam como indicadores de mau prognóstico ${ }^{4,5}$. A tabela 1 mostra os critérios utilizados para caracterizar a malária grave, assim como os requisitos para o seu preenchimento. A malária cerebral, o edema pulmonar, a insuficiência renal aguda, a anemia grave e/ou sangramento constituem as maiores complicações da malária grave. Este paciente apresentava todas essas complicações.

A malária cerebral é a apresentação clínica mais comum e a principal causa de óbito em pacientes com malária grave, com início súbito, após uma crise convulsiva generalizada ou gradual (ao longo de horas ou dias). A definição de malária cerebral envolve a presença de parasitemia por $P$. falciparum e escala de coma de Glasgow (ECG) < 9, com exclusão de outras causas (hipoglicemia, meningite bacteriana, encefalite viral). Do ponto de vista prático, qualquer alteração do estado mental deve ser tratada como malária cerebral6. Quanto mais profundo o coma, pior é o prognóstico. Ao exame, as alterações neurológicas são semelhan- tes à uma encefalopatia simétrica difusa (p. ex.: encefalopatia metabólica), sendo incomum sinais focais ou rigidez de nuca. Apesar da elevada taxa de mortalidade (10\% a 50\%), a maioria dos pacientes não apresenta seqüelas neurológicas após a alta hospitalar.

As complicações pulmonares geralmente ocorrem em poucos dias do início da doença, com rápido desenvolvimento mesmo após início do tratamento antimalárico. O edema pulmonar freqüentemente é não cardiogênico e pode evoluir com SARA ${ }^{7}$. As alterações na radiografia de tórax variam de nódulos confluentes a infiltrados bilaterais difusos e/ou basais.

A insuficiência renal aguda observada em pacientes portadores de malária grave comumente é oligúrica $(<$ $400 \mathrm{~mL} /$ dia) ou anúrica ( $<50 \mathrm{~mL} /$ dia), e pode haver necessidade de diálise temporária ${ }^{8}$.

A ocorrência de hipoglicemia é comum nos pacientes com malária grave. As causas são o consumo de glicose pelo próprio parasita, depleção do glicogênio hepático e hiperinsulinemia induzida pela quinina ou quinidina. Esta complicação pode ser de difícil suspeição já que suas manifestações se confundem com as da malária grave (taquicardia, dispnéia, coma, sudorese, convulsões).

A presença de choque em indivíduos portadores de malária grave geralmente está associada à edema pulmonar, acidose metabólica, sepse e/ou hemorragia maciça. Na maioria dos pacientes, as características

Tabela 1 - Critérios e Indicadores de Prognóstico na Malária Grave

\begin{tabular}{|c|c|}
\hline Malária cerebral & $\begin{array}{l}\text { Coma não atribuído a outra causa, com escala de coma de Glasgow } \leq 9 \text {. O coma deve } \\
\text { persistir por no mínimo } 30 \text { minutos após convulsão generalizada }\end{array}$ \\
\hline Anemia grave & $\begin{array}{l}\text { Hematócrito }<15 \% \text { ou hemoglobina }<5 \mathrm{~g} / \mathrm{dL} \text { na presença de contagem parasitária de }> \\
10000 / \mu \mathrm{L}\end{array}$ \\
\hline Insuficiência renal & $\begin{array}{l}\text { Débito urinário }<400 \mathrm{~mL} / 24 \mathrm{~h} \text { em adultos ( }<12 \mathrm{~mL} / \mathrm{kg} / 24 \mathrm{~h} \text { em crianças) e creatinina sérica } \\
>3 \mathrm{mg} / \mathrm{dL} \text { apesar de reposição volêmica adequada }\end{array}$ \\
\hline Edema pulmonar e SARA & $\begin{array}{l}\text { Relação } \mathrm{PaO}_{2} / \mathrm{FiO}_{2}<200 \text {, associado a infiltrado pulmonar bilateral e pressão de oclusão } \\
\text { de artéria pulmonar }<18 \mathrm{mmHg}\end{array}$ \\
\hline Hipoglicemia & Glicemia $<40$ mg/DI \\
\hline Colapso circulatório & $\begin{array}{l}\text { Pressão arterial sistólica }<70 \mathrm{mmHg} \text { para pacientes }>5 \text { anos de idade }(<50 \mathrm{mmHg} \text { em } \\
\text { crianças de } 1 \text { a } 5 \text { anos) }\end{array}$ \\
\hline Sangramento anormal e/ou CIVD & $\begin{array}{l}\text { Sangramento espontâneo na gengiva, nariz, trato gastrintestinal ou evidência laboratorial } \\
\text { de CIVD }\end{array}$ \\
\hline Convulsões generalizadas de repetição & Três ou mais convulsões num intervalo de $24 \mathrm{~h}$ \\
\hline Acidemia/acidose & $\mathrm{pH}$ arterial $<7,25$ ou acidose $\left(\mathrm{HCO}_{3}^{-}<15 \mathrm{mmol} / \mathrm{L}\right)$ \\
\hline Hemoglobinúria macroscópica & Hemólise que não é secundária à deficiência de G6PD \\
\hline \multicolumn{2}{|l|}{ Alteração do nível de consciência } \\
\hline \multicolumn{2}{|l|}{ Prostração ou fraqueza } \\
\hline Hiperparasitemia & $>5 \%$ de eritrócitos parasitados ou > 250.000 parasitas $/ \mu \mathrm{L}$ \\
\hline Hiperpirexia & Temperatura corporal $>40^{\circ} \mathrm{C}$ \\
\hline Hiperbilirrubinemia & Bilirrubina total $>2,5 \mathrm{mg} / \mathrm{dL}$ \\
\hline
\end{tabular}

SARA = síndrome da angústia respiratória aguda; CIVD = coagulação intravascular disseminada 
do choque são de função cardíaca preservada, com débito cardíaco elevado e resistência vascular periférica baixa.

Como complicações hematológicas, a trombocitopenia é comum, porém geralmente não associada a sangramentos. Cerca de $10 \%$ dos pacientes com malária grave podem apresentar coagulação intravascular disseminada (CIVD). A World Health Organization (WHO) define anemia grave associada à malária como hematócrito menor que $15 \%$ ou hemoglobina menor que 5 $\mathrm{g} / \mathrm{dL}$, na presença de contagem parasitária de maior que 10000/uL e hemácias normocíticas ${ }^{10}$; entretanto, essa referência tem uso limitado, já que esse cutt-off não considera variações como idade ou nível de endemicidade. A definição da WHO é muito especifica; entretanto, pouco sensível. Na prática, a anemia relacionada à malária é definida como hemoglobina ou hematócrito abaixo do limite inferior do esperado para idade, sexo e estado gestacional, na presença de parasitemia por plasmodium de qualquer densidade em áreas endêmicas e anemia grave definida como hemoglobina menor que 5 ou hematócrito menor que 15 na presença de qualquer densidade de parasitemia ${ }^{9}$.

O método padrão para o diagnóstico de malária é o exame da gota espessa do sangue, com visualização do parasita por microscopia óptica. A determinação da espécie é de extrema importância, principalmente nos portadores de malária por $P$. falciparum, pois auxilia na avaliação prognostica. Atualmente, novas tecnologias vêm sendo desenvolvidas com o intuito de agilizar o diagnóstico e o tratamento da doença. Coloração fluorescente com laranja de acridina, imunoensaios e reação em cadeia da polimerase (PCR) são alguns desses métodos, que chegam a alcançar ou até mesmo superar a sensibilidade e a especificidade do exame da gota espessa no diagnóstico de malária ${ }^{10}$.

Vários estudos demonstram que os fatores de risco para malária grave são idade maior que 65 anos, sexo feminino (principalmente quando associado à gravidez), estado não-imune, comorbidades existentes, ausência de profilaxia antimalárica, atraso no tratamento e gravidade da doença na admissão ${ }^{11-13}$. A despeito do uso de profilaxia exercer um efeito protetor sobre a malária grave o paciente deste caso apresentou espectro mais intenso da doença, o que pode ser justificado pelo fato de ter sido feito uso de quimioprofilaxia inadequada. Em regiões de risco para $P$. Falciparum a cloroquina não deve ser usada devido a resistência apresentada por esses parasitas. Nesses casos a profilaxia indicada é mefloquina ( $5 \mathrm{mg} / \mathrm{kg} / \mathrm{semana}$ ) iniciar uma semana antes e interrompendo apenas quatro semanas após a partida da área endêmica. Essa profilaxia é contraindicada em casos de crianças com mecos de $5 \mathrm{~kg}$, gestantes no primeiro trimestre, distúrbios psiquiátricos ou epiléticos e pacientes exercendo atividades que envolvam coordenação e discriminação espacial. Nesses casos deve ser usado doxiciclina.

No que tange ao tratamento desta infecção, existem regimes específicos, de acordo com a espécie do protozoário e com a faixa etária do paciente. No caso da malária grave, o Ministério da Saúde recomenda o uso de derivados de artemisina, por via parenteral, como fármacos de primeira escolha, havendo complementação do esquema com clindamicina, doxaciclina ou mefloquina, por via oral, no final do tratamento ${ }^{14}$. Uma alternativa de tratamento é a quinina por via venosa, com posterior uso da formulação oral deste medicamento ao final do tratamento ${ }^{14}$. É importante salientar que para qualquer caso de malária grave, o tratamento inicial deve ser por via parenteral, e somente quando paciente conseguir tolerar medicação por via oral, é que se deve completar o tratamento por esta via.

\section{CONCLUSÃO}

O caso apresentado correlacionando-se a anamnese com a manifestação clínica do paciente, tratou-se de um caso de malária grave importada, confirmada posteriormente através do exame da gota espessa do sangue periférico. Conforme já descrito, o escore APACHE II na admissão foi de 37 , com risco de óbito de $88 \%$. Os critérios de malária grave preenchidos pelo paciente foram os seguintes: insuficiência renal aguda, SARA, acidose metabólica, alteração do nível de consciência, hemoglobinúria macroscópica, hiperparasitemia e hiperbilirrubinemia.

Apesar da gravidade do quadro e da ocorrência de diversas complicações, algumas inerentes a grave evolução apresentada pelo paciente e sem relação direta com o plasmodium, recebeu o tratamento antimalárico conforme preconizado pelo Ministério da Saúde. Evoluiu sem seqüelas neurológicas, renais, cardiovasculares ou pulmonares sendo de extrema importância o suporte intensivo dispensado ao paciente.

Por fim, a malária deve ser incluída no diagnóstico diferencial de pacientes com febre inexplicável e piora clínica, com história de viagem nos últimos anos para regiões endêmicas da doença. Atrasos no diagnóstico e no tratamento adequado aumentam a morbimortali- 
dade. Todo paciente com malária grave deve ser encaminhado ao CTI, para tratamento de suporte mais adequado. Esse tratamento deve ser feito por via parenteral o mais precocemente possível, baseado nas recomendações do Ministério da Saúde.

\section{REFERÊNCIAS}

01. Muentener P, Schlagenhauf P, Steffen R - Imported malaria (1985-95): trends and perspectives. Bull World Health Organ, 1999;77:560-566.

02. Sachs J, Malaney P - The economic and social burden of malaria. Nature, 2002;415:680-685.

03. Genton B, D'Acremont V - Clinical Features of Malaria in Returning Travelers and Migrants, em: Hamilton SP - Travelers' Malaria. BC Decker; 2001;371-392.

04. Kain KC, Harrington MA, Tennyson S et al - Imported malaria: prospective analysis of problems in diagnosis and management. Clin Infect Dis, 1998;27:142-149.

05. D'Acremont V, Landry P, Mueller I et al - Clinical and laboratory predictors of imported malaria in an outpatient setting: an aid to medical decision making in returning travelers with fever. Am J Trop Med Hyg, 2002;66:481-486.

06. Prakash J, Gupta A, Kumar O et al - Acute renal failure in falciparum malaria--increasing prevalence in some areas of India--a need for awareness. Nephrol Dial Transplant, 1996;11:2414-2416.

07. Ministério da Saúde: Manual de Terapêutica da Malária. 2001.

08. Lee $\mathrm{SH}$, Kara UA, Koay $\mathrm{E}$ et al - New strategies for the diagnosis and screening of malaria. Int J Hematol, 2002;76:(Suppl1):291-293.

09. Menendez, C., A.F. Fleming, and P.L. Alonso, Malaria-related anaemia. Parasitology Today, 2000. 16(11): p. 469-475.

10. Bruneel F, Hocqueloux L, Alberti C et al - The clinical spectrum of severe imported falciparum malaria in the intensive care unit: report of $188 \mathrm{ca}-$ ses in adults. Am J Respir Crit Care Med, 2003;167:684-689.

11. Severe and complicated malaria. World Health Organization. Division of Control of Tropical Diseases. Trans R Soc Trop Med Hyg, 1990;84:(Sup$\mathrm{pl2):S1-S65.}$

12. Severe falciparum malaria. World Health Organization, Communicable Diseases Cluster. Trans R Soc Trop Med Hyg, 2000;94:(Suppl1):S1-S90.

13. Trampuz A, Jereb M, Muzlovic I, Prabhu RM: Clinical review: Severe malaria. Crit Care, 2003;7:315-323.

14. Schwartz E, Sadetzki S, Murad H et al - Age as a risk factor for severe Plasmodium falciparum malaria in nonimmune patients. Clin Infect Dis, 2001;33:1774-1777. 